\title{
TREE-LIKE CONTINUA AND EXACTLY $k$-TO-1 FUNCTIONS
}

\author{
JO HEATH
}

(Communicated by Dennis K. Burke)

\begin{abstract}
To answer a question of Nadler and Ward, $k$-to-1 maps from treelike continua onto tree-like continua are constructed, for $k>2$. It is shown that certain arc-like continua cannot be the domain of any 2-to-1 map and that certain tree-like continua cannot be the image of any 2-to-1 map (defined on continua) but it is unknown if any indecomposable arc-like continuum can be the domain or any tree-like continuum the image of a 2-to-1 map.
\end{abstract}

\section{INTRODUCTION}

In [11], Nadler and Ward prove that any continuum not hereditarily unicoherent is the exactly $k$-to-1 continuous image of some continuum and they prove that any continuum whose every subcontinuum has an endpoint cannot be the exactly $k$-to-1 continuous image of any continuum, unless $k=1$. In [4] it was shown that no dendrite is the (exactly) $k$-to-1 image of any continuum even if the function is allowed to be finitely discontinuous. These results leave unanswered a question that Nadler and Ward ask, namely can any tree-like continuum be the (exactly) $k$-to-1 image of a continuum? Constructed in this paper (in $\S I$ ) is an example of an arc-like continuum that admits a $k$-to-1 continuous map onto itself for any odd integer $k$, and for each even integer $k>2$, two tree-like continua are constructed and a $k$-to- 1 map from one onto the other.

The difficult case is $k=2$. Two settings are studied: 2-to-1 functions with arc-like domains in §II and 2-to-1 functions with tree-like images in §III.

For the case of the tree-like domain of a 2-to-1 map there are easy examples, one from [1] is illustrated on the next page.

Also, Wayne Lewis has constructed a 2-to-1 map on an arc-like continuum, see Example 3 in $\S I I$, but the following question is not yet answered.

Question 1. Can any indecomposable arc-like continuum be the domain of a 2-to-1 continuous map?

Received by the editors November 24, 1987 and, in revised form, February 12, 1988.

1980 Mathematics Subject Classification (1985 Revision). Primary 54C10; Secondary 26A03, $26 \mathrm{~A} 15$

Key words and phrases. $k$-to-1 function, 2-to-1 function, tree-like continua.

Presented, in part, at the Atlanta AMS meeting, January 1988. 


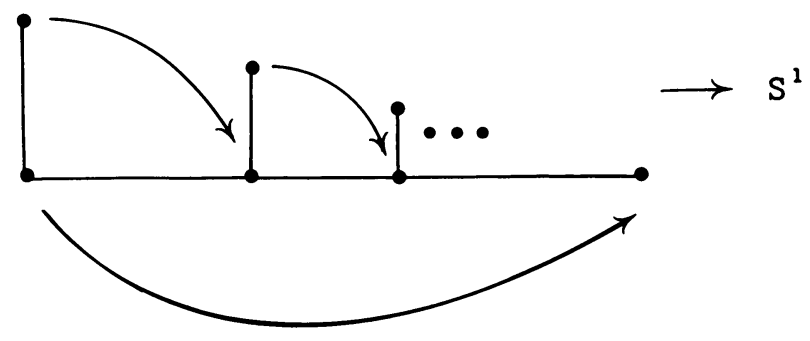

Figure 1. "An example of a 2-to-1 map on a tree-like continuum"

In [9, (IV, 4 and 5)], Mioduszewski showed that neither the arc-like indecomposable Knaster bucket-handle continuum (described in Example 1 in $\S I I$ ) nor the arc-like decomposable Knaster $V$-continuum (see [6], p. 570) can be the domain of a 2-to-1 map and in §II, the class of continua which cannot be the domain of a 2-to-1 finitely discontinuous function is shown to include all finitely discontinuous 1-to-1 images of $[0,1]$, and these continua are shown to be tree-like.

In §III, which concerns tree-like images, an example of a 2-to-1 finitely discontinuous function from a tree-like continuum onto the arc-like Knaster buckethandle space is given, but no example is known for continuous maps.

Question 2. Can any tree-like continuum be the 2-to-1 continuous image of a continuum?

Some tree-like continua can be ruled out. It is shown here that no 1-to1 finitely discontinuous image of $[0,1]$, necessarily tree-like, can be such a continuous image, nor any continuum whose every subcontinuum has a finite separating set. More generally, it is shown that if every subcontinuum of a continuum $Y$ "can be pruned" (a generalization of "has a cut point") then $Y$ is not the continuous 2-to-1 image of any continuum.

All continua are understood to be compact and metric.

\section{$\S \mathbf{I}$}

The examples in this section answer affirmatively for all $k>2$ the NadlerWard question: can a tree-like continuum be the $k$-to- 1 image of some continuum? Exactly which tree-like continua are images, however, is not known.

Example 1. An arc-like continuum $X$ that maps $k$-to-1 onto itself for each odd positive integer $k$. 
The function used will be a union of sawtooth functions as follows. If $k$ is a positive odd integer and $A$ is an arc, choose $k+1$ points of $A, a_{1}<a_{2}<$ $\cdots a_{k+1}$, with $a_{1}$ and $a_{k+1}$ endpoints of $A$, and define a continuous $f$ on $A$ so that $f\left(a_{1}\right)=a_{1}, f\left(a_{k+1}\right)=a_{k+1}$, and for each $i$ from 1 to $k, f$ restricted to the subarc $\left[a_{i}, a_{i+1}\right]$ is a homeomorphism onto $A$. Note that $f$ is $k$-to-1 at each interior arc point, $f$ is $[(k+1) / 2]$-to- 1 at each endpoint of $A$, and if $A$ and $B$ are two arcs sharing only a common endpoint $x$ with sawtooth functions defined on each, then the union of the functions is $k$-to- 1 at $x$.

Let $Y$ denote the classic planar Knaster bucket-handle space (see [7]) constructed as follows. Let $C$ denote the usual deleted middle thirds Cantor set in $[0,1]$ and let $Y=\bigcup S_{i}$, where $S_{1}$ is the union of all semicircles in $\{y \geq 0\}$ with center $\left(\frac{1}{2}, 0\right)$ and both endpoints in $C$, and for $i \geq 3, S_{i-1}$ is the union of all semicircles in $\{y \leq 0\}$ with center $5 /\left(2 \cdot 3^{i}\right)$, diameter no more than $1 / 3^{i}$, and both endpoints in $C$.

Define $X$ to be $Y \cup V$, where $V$ is $Y$ reflected about the $y$-axis. The $k$-to-1 continuous map $f$ from $X$ onto $X$ is simply the union of sawtooth functions defined on each semicircle described in the construction of $Y$, and on each reflected semicircle in $V$. The sawtooth functions need to be defined uniformly on each $S_{i}$ collection of semicircles for $f$ to be continuous.

Example 2. If $k$ is an even integer greater than two, then there are tree-like continua $W$ and $Z$ and a $k$-to-1 continuous map from $W$ onto $Z$.

Let $X=Y \cup V$ be the continuum described in Example 1. To construct $W$, glue the $(0,0)$ points of $(k-3)+(k-3)+(k-2)(k-3)$ copies $V_{1}, \ldots, V_{k-3}$, $Y_{1}, \ldots, Y_{k-3}, Y(1,1), \ldots, Y(k-2, k-3)$ of $Y$ to $X$ at $(0,0)$. For each $\varepsilon>0, W$ can be $\varepsilon$-mapped onto an $n$-od with $n=2+k(k-3)$ and so in particular, $W$ is tree-like. The image space $Z$ is the subspace $X \cup Y_{1} \cup \cdots \cup Y_{k-3}$ of $W$.

Define the $k$-to-1 map $g$ from $W$ onto $Z$ as follows. On $X, g$ is the same as $f$ in Example 1 for $k=3$. On each $V_{i}, g$ is the natural 1-to-1 projection onto $V$, and on each $Y(i, j), g$ is the natural 1-to-1 projection onto $Y_{i}$ if $1<i<k-3$ and onto $Y$ if $i=k-2$. Finally, $g \mid Y_{i}$ maps onto $Y_{i}$, for $1 \leq i \leq k-3$, exactly as $f$ does from $Y$ onto $Y$. It is straightforward to count the $k$ point inverses for each point in $Z$.

\section{$\S$ III}

The collection of 1-to-1 finitely discontinuous images of $[0,1]$ includes, besides arcs and acyclic finite graphs, and $\sin \left(\frac{1}{x}\right)$ curve plus its limiting set, a triod plus a ray spiraling down on the triod, and many other relatively simple continua. All 1-to-1 finitely discontinuous images of $[0,1]$, if continua, are shown to be tree-like in Theorem 1. It is an immediate corollary to the reference theorem below that no 1-to-1 finitely discontinuous image of $[0,1]$ can be the domain of any 2-to-1 finitely discontinuous function onto a Hausdorff space. 
Reference Theorem (Heath, [5]). There is no 2-to-1 finitely discontinuous function defined on $[0,1]$ whose image is Hausdorff.

Until recently there were no known examples of 2-to-1 maps defined on chainable continua but the following is a new result of Wayne Lewis.

Example 3 (I. W. Lewis). A chainable continuum $X$ that admits an exactly 2-to-1 map onto a continuum.

Construction. Lewis has shown in [8] that for each $n \geq 2$ there is a chainable continuum $C$ admitting a homeomorphism $h$ of period $n$ such that $h$ has exactly one fixed point $p$ and every other point of $C$ has minimal period $n$. Furthermore, there is a point $q$ in $C$ such that for each $\varepsilon>0$ there is an $\varepsilon$-chain covering $C$ with $p$ and $q$ in opposite end-links. The continuum $C$ will be indecomposable and may be chosen to be the pseudo-arc.

For $i=1,2,3, \ldots$ let $C_{i}$ denote a copy of $C$ for $n=2$ with diameter less than $1 / 2^{i}$, and let $p_{i}$ and $q_{i}$ denote the copies of $p$ and $q$ respectively in $C_{i}$. To construct $X$, string together the $C_{i}$ by identifying $q_{i}$ and $p_{i+1}$ for $i=1,2, \ldots$, and add a limiting point, $p_{w}$, of $\left\{C_{i}\right\}$. Consider the space $X^{\prime}$ obtained from $X$ by identifying each point $x$ of $C_{i}$ with $h_{i}(x)$ (where $h_{i}$ is the period 2 homeomorphism of $C_{i}$ with fixed point $p_{i}$ ) and also identifying $p_{1}$ with $p_{w}$. Then there is a 2-to-1 map from $X$ onto $X^{\prime}$, namely the map determined by the identifications.

Theorem 1. If the continuum $Y$ is a 1-to-1 finitely discontinuous image of $[0,1]$, then $Y$ is tree-like and each subcontinuum of $Y$ has a cut-point.

Proof. If $f:[0,1] \rightarrow Y$ for some continuum $Y$, where $f$ is 1-to-1 and finitely discontinuous, certain "end" sets in $y$ can be defined. If $(a, b)$ is a component of $(0,1)$ minus the discontinuities and $c$ is in $(a, b)$, then $\operatorname{End}(a, c)$ denotes those points in $Y$ that are limits of some $\left\{f\left(x_{i}\right)\right\}$ sequence, where $x_{i}$ is in $(a, c)$ and $\left\{x_{i}\right\} \rightarrow a$. Similarly $\operatorname{End}(c, b)$ is defined. Each end is compact and connected.

Now suppose there are continua that satisfy the hypothesis but not the conclusion, and let $Y$ denote one of the counter-examples with the least number of nondegenerate ends, let $f$ denote the corresponding 1-to-1 finitely discontinuous function and let DIS denote the set of discontinuities of $f$ plus the endpoints $\{0,1\}$.

Case 1. Suppose $Y$ has no nondegenerate end. Then for each component $(a, b)$ of $[0,1]-$ DIS, $\overline{f((a, b))}$ is a finite graph and so $Y$ is a graph. Let $J$ denote $f(\mathrm{DIS})$ plus the (degenerate) ends in $Y$. Then each component of $[0,1]-f^{-1}(J)$ maps onto a component of $Y-J$. Suppose $[0,1]-f^{-1}(J)$ has $m$ components. Then $m=\left|f^{-1}(J)\right|-1=|J|-1$. Since $f$ is 1-to-1, $Y-J$ has $m$ components also. Consider $J$ as the vertices of the graph $Y$. Then the 
Euler number of $Y$ is $m-|J|=-1$ and so $Y$ has no simple closed curve. Each acyclic graph satisfies the conclusion of the theorem, so case 1 cannot hold.

Case 2. Suppose $Y$ has at least one nondegenerate end. We will show that for some component $(a, b)$ of $[0,1]$ - DIS and some $\delta>0$, either $f((a, a+\delta))$ or $f((b-\delta, b))$ is open in $Y$.

Claim. Suppose $\left\{x_{i}\right\} \rightarrow x_{0} \in$ DIS, each $x_{i}<x_{0}$, and $M$ is a continuum in $Y$ containing each $f\left(x_{i}\right)$. Then there is an $\varepsilon>0$ such that $M$ contains $f\left(\left(x_{0}-\varepsilon, x_{0}\right)\right)$.

First note that if $K$ is a continuum in $Y,[a, b] \subset I$ - DIS and neither $f(a)$ nor $f(b)$ belongs to $K$ but $f(c)$ is in $K$ for some $c$ in $(a, b)$, then either $K \subset f([a, b])$ or for some discontinuity $x$ and some $\delta>0, K$ contains either $f((x, x+\delta))$ or $f((x-\delta, x))$. Otherwise there are at least two but no more than countably many disjoint closed intervals $I_{1}, I_{2}, \ldots$ in $[0,1]-$ DIS such that $K$ is the union of countably many disjoint compacta $f(\mathrm{DIS}) \cap K$ and $f\left(I_{j}\right) \cap K, j=1,2, \ldots$. This contradicts Theorem $56[9$, p. 23].

Now suppose $\left\{x_{i}\right\}, x_{0}$, and $M$ satisfy the hypothesis of the claim.

If no such $\varepsilon$ exists, then there is another sequence $\left\{x_{i}^{\prime}\right\} \rightarrow x_{0}$ such that for each $i, x_{i}^{\prime}<x_{i+1}^{\prime}<x_{0}, f\left(x_{i}^{\prime}\right)$ is not in $M$, and some $x_{j}$ lies between $x_{i}^{\prime}$ and $x_{i+1}^{\prime}$. For each integer $n$ there are open sets $U_{1}, \ldots, U_{n}$ in $M$ with disjoint closures such that $U_{i}$ contains the compactum $f\left(\left[x_{i}^{\prime}, x_{i+1}^{\prime}\right]\right) \cap M$ for $i=1,2, \ldots, n$. Since the continuum $M$ is not in $U_{1}$, the component $L_{1}$ of $U_{1}$ containing some $f\left(x_{j}\right)$ for $x_{1}^{\prime}<x_{j}<x_{2}^{\prime}$ has a limit point $p_{1}$ in $M$ on the boundary of $U_{1}$ ("to the boundary" theorem, for instance in [7]). Since $\bar{L}_{1}$ contains neither $f\left(x_{1}^{\prime}\right)$ nor $f\left(x_{2}^{\prime}\right)$ but does contain $f\left(x_{j}\right)$ and a point $p$ not in $f\left(\left[x_{1}^{\prime}, x_{2}^{\prime}\right]\right)$ it follows from the earlier observation that $\bar{L}_{1}$ contains an end. Similarly, each $\bar{U}_{j}$ contains an end, contradicting the fact that there are only finitely many ends in $Y$.

Now, let $E$ denote the union of all the nondegenerate ends in $Y$. Write $[0,1]-\mathrm{DIS}=C_{1} \cup C_{2} \cup \cdots$, where each $C_{i}$ is a closed interval. Then $E$ is the countable union of the closed sets $E \cap f(\mathrm{DIS}), E \cap f\left(C_{1}\right), E \cap f\left(C_{2}\right), \ldots$. Since $E-f(\mathrm{DIS})$ is locally compact, it satisfies the Baire category theorem, so some $p$ in some $E \cap f\left(C_{j}\right)$ is not a limit point of the union of the other compacta. Since $p \in E, p$ belongs to a nondegenerate end, say $\operatorname{End}(x-\delta, x)$. Thus there is a sequence $\left\{x_{i}\right\} \rightarrow x, x_{i}<x$, with $\left\{f\left(x_{i}\right)\right\} \rightarrow p$ and each $x_{i} \notin E$. Thus no end contains $f((x-\varepsilon, x))$ for any $\varepsilon>0$ and it follows from the claim (since each end is a continuum) that for each end there is an $\varepsilon^{\prime}$ so that the end misses $f\left(\left(x-\varepsilon^{\prime}, x\right)\right)$. There are only finitely many ends, degenerate or nondegenerate, so some $\delta^{\prime}>0$ exists such that no point of $f\left(\left(x-\delta^{\prime}, x\right)\right)$ belongs to any end.

Since $f\left(\left(x-\delta^{\prime}, x\right)\right)$ is open in $Y$, its complement is compact. Let $Y^{\prime}=$ $Y-f\left(\left(x-\delta^{\prime}, x\right)\right) \cup A$, where $A$ is an open arc added to $Y$ so that the endpoints 
of $A$ are $q$ and $q^{\prime}=f\left(x-\delta^{\prime}\right)$. Then $Y^{\prime}$ is a continuum. Now define $\hat{f}$ from $[0,1]$ onto $Y^{\prime}$ by $\hat{f}=f \mid\left([0,1]-\left(x-\delta^{\prime}, x\right)\right) \cup f_{1}$ where $f_{1}$ is a homeomorphism from $\left[x-\delta^{\prime}, x\right)$ onto $\bar{A}-\{q\}$. Then $\hat{f}$ is also 1-to-1 and finitely discontinuous, and $\hat{f}$ has one less (than $f$ ) nondegenerate end in $Y^{\prime}$ so $Y^{\prime}$ is tree-like by assumption.

Note that $Y^{\prime}-A=H \cup K$, two disjoint continua, since no component of $Y^{\prime}-A$ can have both $q$ and $q^{\prime}$ in its closure (because $Y^{\prime}$ is tree-like and hence unicoherent). This means that $Y-f\left(\left(x-\delta^{\prime}, x\right)\right)=H \cup K$, so $Y$ is the union of two disjoint tree-like continua $H \cup K$ plus a ray from one to the other, and $Y$ must be tree-like also.

If $M$ is a subcontinuum of $Y$ that intersects $f\left(\left(x-\delta^{\prime}, x\right)\right)$, then each interior point of $f\left(\left(x-\delta^{\prime}, x\right)\right) \cap M$ is a cut point of $M$ and if $M \subset H$ or $M \subset K$ then $M$ has a cut point since each subcontinuum of $Y^{\prime}$ has a cut point by assumption.

\section{§III}

Nadler and Ward showed in [11] that if each subcontinuum of $Y$ has an endpoint then $Y$ is not the $k$-to-1 image of any continuum, and in [1] D. Fox proved that if each subcontinuum of $Y$ has a cut point then $Y$ is not a 2to-1 continuous image of any continuum (see Corollary 1 ). As a direct result (Corollary 2), it follows from Theorem 1 that no 1-to-1 finitely discontinuous image of $[0,1]$ can be the 2-to-1 image of any continuum. The class of treelike continua which are known not to be 2-to-1 images is further expanded in Corollary 3 and Theorem 3 to include continua whose subcontinua either have finite separating sets or can be "pruned". An example of a tree-like continuum which can be pruned but has no end point nor finite separating set is the Cantor parquet set, CP:

Let $C$ be the Cantor set in $[0,1]$ and define

$$
\begin{aligned}
\mathrm{CP}= & \{(x, y): x \in C, 0 \leq y \leq 1\} \cup\{(x, y): y \in C,-1 \leq x \leq 0\} \\
& \cup\{(x, y):-x \in C,-1 \leq y \leq 0\} \cup\{(x, y):-y \in C, 0 \leq x \leq 1\} .
\end{aligned}
$$

And lastly, Example 4 shows there is a tree-like continuum, in fact an arc-like continuum, that is the 2-to-1 finitely discontinuous image of a continuum.

Reference Theorem (D. Fox [1, paraphrase of Theorems 1 and 3]). If $f$ maps the continuum $X$ onto the continuum $Y$ then there is a subcontinuum $Y^{\prime}$ of $Y$ such that (1) $f^{-1}\left(Y^{\prime}\right)$ is a continuum but for no proper subcontinuum $Y^{\prime \prime}$ of $Y^{\prime}$ is $f^{-1}\left(Y^{\prime \prime}\right)$ a continuum, and (2) if $Y^{\prime}$ satisfies (1) and the point $p$ separates $Y^{\prime}$ into $n$ disjoint separated sets, then $f^{-1}(p)$ has more than $n$ components.

Corollary 1 (Also proved in [2] and in [10]). If each subcontinuum of the continuum $Y$ has a cut point, then $Y$ is not the 2-to-1 continuous image of any continuum.

Corollary 2. If $Y$ is the 1-to-1 finitely discontinuous image of $[0,1]$ then $Y$ is not the 2-to-1 continuous image of any continuum. 
Corollary 3. If each subcontinuum of the continuum $Y$ has a finite separating set and $Y$ is hereditarily unicoherent, then $Y$ is not the 2-to-1 image of any continuum.

Proof. Suppose $M$ is a subcontinuum of $Y$ and the finite set $F \subset M$ minimally separates $M$. Then $M-F=U \cup V$, two disjoint open sets. If the point $p$ of $F$ belongs to $\bar{U}-U$ and does not belong to $\bar{V}-V$ then $M-(F-\{p\})=(U \cup\{p\}) \cup V$, two disjoint open sets. This contradicts the minimality of $F$. Hence $F=[\bar{U}-U] \cap[\bar{V}-V]=\bar{U} \cap \bar{V}$. Now suppose $\bar{U}$ is not connected, $\bar{U}=A \cup B$, two separated closed sets. Since $M$ is connected and $M=A \cup(B \cup V)=B \cup(A \cup V), A$ and $B$ both intersect $F$. But $M-(F \cap A)=[A-F] \cup[V \cup B]$, two separated sets and $F \cap A$ is a proper subset of $F$ (since $F \cap B$ exists). Hence $\bar{U}$ is connected, and likewise $\bar{V}$. Since $m$ is unicoherent and $\bar{V} \cap \bar{U}$ is $F, F$ must be a single point. Thus each subcontinuum of $Y$ has a cut point and Corollary 3 follows from Corollary 1 .

Definition. The closed, totally disconnected set $K$ in the nondegenerate continuum $Y$ prunes $Y$ if $Y-K$ is disconnected and every component of $Y-K$ except one has exactly one point of $K$ in its closure.

Lemma. If $X$ and $Y$ are continua, $f: X \rightarrow Y$ is 2-to-1 and continuous and if some set $K$ prunes $Y$, then some proper subcontinuum of $Y$ has connected inverse.

Proof. Let $D$ be a component of $Y-K$ with one point, $p(D)$, of $K$ in its closure. If some component of $X-f^{-1}(K)$ that maps into $D$ has both points of $f^{-1}(p(D))$ in its closure then $f^{-1}(\bar{D})$ is connected. Hence we may assume that each component $V$ of $X-f^{-1}(K)$ with $f(V) \subset D$ has only one point, $q(V)$, of $f^{-1}(p(D))$ in its closure.

Since $Y$ is connected, some component $W$ of $Y-K$ has $K$ in its closure. Either $f^{-1}(\bar{W})$ is connected and the theorem is proved, or $f^{-1}(\bar{W})=$ $N \cup M$, two disjoint closed sets in $X$. Since $k$-to- 1 maps preserve dimension [3], $f^{-1}(K)$ is also closed and totally disconnected, so there is a disconnection $f^{-1}(K)=K_{1} \cup K_{2}$ so that $N \cup K_{1}$ and $M \cup K_{2}$ are disjont closed sets. Define $N^{\prime}=N \cup K_{1} \cup\left(\cup\left\{V: q(V) \in K_{1}\right.\right.$ and $\left.\left.V \in \mathscr{V}\right\}\right)$ and $M^{\prime}=M \cup K_{2} \cup\left(\bigcup\left\{V: q(V) \in K_{2}\right.\right.$ and $\left.\left.V \in \mathscr{V}\right\}\right)$, where $\mathscr{V}$ is the collection of components of $X-f^{-1}(K)$ whose image has only one limit point in $K$. Then $X=N^{\prime} \cup M^{\prime}$ is a disconnection of $X$, a contradiction.

Theorem 2. If every subcontinuum of the continuum $Y$ can be pruned then $Y$ is not the 2-to-1 continuous image of any continuum.

Proof. Theorem 2 follows directly from the Lemma and part (1) of the D. Fox reference theorem.

Example 4. There is a 2-to-1 function with only one discontinuity from a hereditarily decomposable tree-like continuum onto the indecomposable arclike Knaster bucket-handle space (described in Example 1). 
Let $F(1), F(2), \ldots$ denote disjoint copies of a Cantor fan such that for each $i, F(i)$ has diameter less than $1 / i$ and $x_{i}$ denotes its only cut point. (A Cantor fan is a copy of $C \times[0,1]$ with the points of $C \times\{1\}$ identified, where $C$ is a Cantor set.) Let $W$ be the union of the $F(i)$ with the $x_{i}$ points identified as a single point $x$. The point $x$ will be the only discontinuity for $g: W \rightarrow Y$, where $Y$ is the bucket-handle space. Each $F(i)-\{x\}$ is a copy of $C \times[0,1)$, and recall that $Y=\bigcup S_{i}$ (see Example 1) where $S_{i}$ is a collection of semicircles homeomorphic to $C \times[0,1]$. Let $f_{i}$ denote a homeomorphism from $C \times[0,1]$ onto $S_{i}$, for each $i$, and let $h$ denote the reversing homeomorphism from $C \times[0,1)$ onto $C \times(0,1]$ defined by $h(c, r)=(c, 1-r)$. Now define $g_{i}$ from $(F(2 i) \cup F(2 i-1))-\{x\}$ onto $S_{i}$ by $g_{i}(p)=f_{i}(p)$ if $p$ is in $F(2 i)$, and $g_{i}(p)=f_{i} h(p)$ if $p$ is in $F(2 i-1)$. Note that $g_{i}$ is 1-to-1 at the points of $S_{i}$ on the $x$-axis, i.e. on $f_{i}^{-1}(C \times\{0,1\})$, and $g_{i}$ is 2-to-1 at the other points of $S_{i}$. Finally, let $g$ be the union of the $g_{i}$ maps plus the ordered pair $(x,(0,0))$.

\section{REFERENCES}

1. D. Fox, $k$-to-1 continuous transformations, University of California at Riverside Dissertation, August 1973.

2. Gottschaulk, On k-to-1 transformations, Bull. Amer. Math. Soc. 53 (1947), 168-169.

3. O. G. Harold, The non-existence of a certain type of continuous transformation, Duke Math. J. 5 (1939), 789-793.

4. J. Heath, There is no $k$-to-1 function from any continuum onto $[0,1]$, or any dendrite, with only finitely many discontinuities, Trans. Amer. Math. Soc. 306 no. 1 (1988), 293-305.

5. __, Every exactly 2-to-1 function on the reals has an infinite number of discontinuities, Proc. Amer. Math. Soc. 98 (1986), 369-373.

6. B. Knaster, Un continu irreductible a decomposition contiue en tranches, Fund. Math. 25 (1935), p. 568-577.

7. C. Kuratowski, Topologie, I (1948) and II (1950), Warszawa-Wroclaw.

8. I. W. Lewis, Periodic homeomorphisms of chainable continua, Fundamenta Mathematicae 117 (1983), 81-84.

9. J. Mioduszewski, On two-to-one continuous functions, Dissertationes Math. (Rozprawy Mat.) 24 (1961), 42.

10. Robert L. Moore, Foundations of point set theory, Amer. Math. Soc. Colloq. Publ., vol. 13, Amer. Math. Soc., Providence, R.I., 1962.

11. S. B. Nadler, Jr and L. W. Ward, Jr., Concerning exactly $(n, 1)$ images of continua, Proc. Amer. Math. Soc. 87 (1983), 351-354.

Foundations, Analysis and Topology and Mathematics Departments, Auburn UniverSITY, AUBURn University, Alabama 36849-5310 